\title{
Identification of Tumor Specific Neoantigens and Their Implications in Dendritic Cell Immunotherapy Using Liquid Biopsy Results: Findings from an Observational Cohort Study
}

\section{Khin Zay Yar Myint}

Center for Advanced Medical Science and Technology, Tokyo Midtown Medical Center https://orcid.org/0000-0001-5553-1245

Masamori Shimabuku

Center for Advanced Medical Science and Technology, Tokyo Midtown Medical Center Ruriko Horio

Center for Advanced Medical Science and Technology, Tokyo Midtown Medical Center Munehisa Kaneda

Center for Advanced Medical Science and Technology, Tokyo Midtown Medical Center

Yoko Shimizu

Center for Advanced Medical Science and Technology, Tokyo Midtown Medical Center Junichi Taguchi ( $\nabla_{\text {j-taguchi@tokyomidtown-mc.jp ) }}$

Center for Advanced Medical Science and Technology, Tokyo Midtown Medical Center

\section{Research Article}

Keywords: Liquid biopsy, genetic profiling, neoantigens, dendritic cell immunotherapy

Posted Date: December 6th, 2021

DOI: https://doi.org/10.21203/rs.3.rs-1069498/v3

License: (c) (i) This work is licensed under a Creative Commons Attribution 4.0 International License. Read Full License 


\section{Abstract}

Liquid biopsies can be a rapid, cost-effective and noninvasive alternative to tumor biopsies for detecting genetic mutations in somatic tumors. Genetic profiling of liquid biopsies can also be used to identify novel antigens for targeted therapy, provide updated information on disease prognosis and evaluate treatment efficacy. In this study, we aimed to examine mutations that could be identified in liquid biopsy and their potential implications for personalized dendritic cell immunotherapy using these antigens. We analyzed the genomic profiles of 99 blood samples from 85 patients with 22 different types of cancer using two commercially available liquid biopsy tests before the patients underwent standard cancer treatment and dendritic cell immunotherapy. Nonsynonymous mutations were detected in more than $90 \%$ of the samples, with an average frequency of 3.6 mutations per sample. The tumor mutations were specific to each patient, as approximately $94.7 \%$ of the mutations were so unique that there was almost no duplication among the patients. Clonal evolution was observed in two patients just before or after chemotherapy, radiotherapy and immunotherapy. These findings indicate that liquid biopsy can be a potential surrogate for tumor-specific antigen-based immunotherapy and the importance of tailoring immunotherapy in accordance with the liquid biopsy result in each treatment stage.

\section{Introduction}

With the use of tumor genomic profiling technology, tissue biopsy can provide a vast amount of genetic information on genomic alterations. However, the turn-around time to obtain such results is significantly long. On the other hand, liquid biopsy, which is the analysis of free circulating tumor DNA (ctDNA) from blood or plasma, can detect genomic alterations within a much shorter time without requiring invasive sampling. According to a study by Cancer Center Japan, liquid biopsy can shorten the turn-around time by 22 days and help more patients enroll in eligible clinical trials compared to conventional tissue biopsy [1].

Since cancers evolve through repeated mutation of oncogenic clones, subclonal and emerging mutations can be overlooked in genomic profiling of tissue biopsies, whereas liquid biopsy can reflect real-time genomic profiles. In addition, tissue biopsy can only reflect the genomic profile of a part of the tumor, not the whole tumor mass. Furthermore, tissue biopsy involves invasive procedures, limiting its ability for repeat testing in cases in which the sample is insufficient, there are multiple metastatic sites, or serial testing is desired to monitor disease progression. In contrast, liquid biopsy saves time and is less invasive than and noninferior to conventional tissue biopsy. Liquid biopsy can reflect some of the emerging resistant mutations and may include DNA shed from different metastatic sites.

Sunami et al. reported that although $59.4 \%$ of patients had actionable gene aberrations in their genomic profiling results of tissue biopsies, only $13.3 \%$ received molecular-targeted therapy based on their results [2]. This large gap indicates the need to apply tumor genomic information in applications beyond molecular targeted therapy. One such potential area is personalized cancer immunotherapy. One challenge for personalized immunotherapy is the availability of tumor biopsies. Tumor-specific antigens 
are usually identified from genomic profiling of tumor biopsies. However, this conventional method is time-consuming, invasive, incapable of capturing clonal evolution and unfeasible in cases in which repeated sampling is required or there is insufficient sample, which are more common with recurrent and metastatic tumors. Therefore, liquid biopsies can be a good surrogate for discovering tumor-specific antigens as potential targets for immunotherapy. Although there is limitation in the detection of genomic mutations with very low allele frequency in plasma, reliability of genetic information has been reported regarding the use of liquid biopsy [3-5]. Thus, liquid biopsies might provide valuable insights for making treatment decisions.

In this study, we aimed to examine tumor-specific antigens that can be identified in liquid biopsy and how such antigens can aid the development of personalized dendritic cell immunotherapy.

\section{Materials And Method}

\section{Study population}

This study was a single-center, cross-sectional study conducted in 85 patients who consulted for dendritic cell immunotherapy at the Department of Advanced Medical Science and Technology, Tokyo Midtown Medical Center. Twelve patients underwent genomic testing twice, and one patient underwent genomic testing three times. We analyzed the results of 99 samples in total.

\section{Ethical approval and informed consent}

The study was approved by the Institutional Review Board of Midtown Clinic Medical Corporation. Prior to the blood tests, we explained the nature of the genomic profiling tests, including their benefits, risks, limitations, and utilization of their results in clinical research. We also informed the participants of their right to withdraw anytime and obtained written informed consent upon administration of liquid biopsy testing.

\section{Comprehensive genomic profiling using liquid biopsy}

We used two commercially available genomic profiling tests to assess cell-free DNA in the patients' plasma: Guardant360 (Guardant Health, Redwood City, CA, USA) and GenoDive (GenoDive Pharma, Atsugi, Kanagawa, Japan). The Guardant360 assays were performed at a Clinical Laboratory Improvement Act-certified, College of American Pathologists-accredited laboratory. The assay uses nextgeneration sequencing technology to identify single-nucleotide variants (SNVs) and deletion variants in 74 genes, copy-number amplifications in 18 genes, and fusions in 8 genes (Supplementary data file 1). The genomic profiling results of the prospective analysis of 194 advanced cancer patients using the 
Guardant360 assay effectively guided treatment selection, and response rates to those selected treatments were comparable to those of tissue biopsy-guided therapies [6].

In contrast, GenoDive assays were performed at the GenoDive Pharma Okinawa Branch, where plasma separation was performed according to Streck's instructions (Streck Inc., La Vista, NE, USA), and cfDNA purification was performed using a QIAamp circulating nucleic acid purification kit (Qiagen). Tumor profiling was performed at the laboratory using the AVENIO ctDNA Expanded Kit (Roche Sequencing Solutions, Pleasanton, CA), which employed integrated digital error suppression (iDES) technology for genotyping and detection of ctDNA [7], using HiSeq (Illumina) next-generation sequencing technology. The primary data analyses were performed by AVENIO ctDNA Analysis Software installed in the Roche Oncology Analysis Server (Roche Diagnostics). The secondary data analysis was performed in-house, mostly based on the duplex-sequencing principle [8]. The AVENIO tumor profiling panel identifies SNVs, insertions and deletions, fusions and copy-number variants in 77 genes, including those currently in the National Cancer Comprehensive Network (NCCN) database and emerging biomarkers investigated in clinical trials (Supplementary data file 2). Studies have demonstrated that AVENIO ctDNA kits are a viable alternative for detecting somatic mutations in clinical laboratories $[9,10]$. All the methods and procedures were carried out in accordance with relevant guidelines and regulations.

\section{Statistical methods}

All continuous variables are expressed as the means and standard deviations. All categorical variables are expressed as numbers and proportions. The calculations and figure generation, except for the generation of the heatmap, were performed using Microsoft Excel (Microsoft Corporation, 2018). The heatmap was generated using R software (R 4.1.0, R Core Team, 2021) and the ComplexHeatmap package [11].

\section{Results}

\section{Patient characteristics}

The study cohort encompassed 99 blood samples from 85 patients with 21 different tumor types. The tumor types included non-small-cell lung cancer (NSCLC, $n=17)$, pancreatic cancer $(n=14)$, breast cancer $(n=12)$, stomach cancer $(n=12)$, colorectal cancer $(n=10)$, biliary cancer $(n=6)$, uterine cancer $(n=4)$, ovarian cancer with peritoneal carcinomatosis $(n=4)$, head and neck cancer $(n=3)$, cervical cancer $(n=3)$, sarcoma $(n=2)$, small-cell lung cancer $(n=2)$, and pancreatic neuroendocrine tumor $(n=2)$, and the remaining 8 cancer types were apocrine gland cancer, tongue cancer, duodenal gastrointestinal stromal tumor, kidney cancer, prostate cancer, pulmonary neuroendocrine tumor, brain tumor and ureteral cancer. The demographic, clinical and pathological characteristics of the study cohort are summarized in Table 1. 


\section{Liquid biopsy reports}

Notably, all 99 samples showed at least one type of alternation in the observed genes. Table 2 shows a summary of the genomic profiling results of the study cohort. The mean circulating free DNA (cfDNA) concentration was $162.7 \pm 352.3$ nanograms per 20 milliliters. In the cfDNA, the circulating tumor DNA percentage ranged from $0.006 \%$ to $90.6 \%$. There were 6 samples with presumed germline pathogenic variants, comprising approximately $6 \%$ of the total cases. A variant was presumed to be a germline mutation if its allele frequency (variant allele frequency or VAF) was approximately $50 \%$ [12]; the range is typically between $40 \%$ and $60 \%$ according to the American College of Medical Genetics and Genomics (ACMG) [13]. With the exception of samples with gene amplification and high microsatellite instability (MSI-high), the number of mutations in each sample varied from zero to 21, with an average of 5.6 mutations in each patient. Among these mutations, nonsynonymous mutations, where a change in one base of the DNA sequence resulted in amino acid changes, were the most frequently observed type of mutation. These mutations were observed in 90 out of 99 samples, with an average frequency of 3.6 mutations per patient. Other types of mutations included synonymous substitution (a change in one base of the DNA sequence not resulting in amino acid changes), frameshift mutation, nonsense mutation, mutation in 5'- and 3'- untranslated regions, partial deletion, fusion, duplication, and gene amplification with MSI-high. The frequency of occurrence of these types of mutations compared to the total number of mutations was $16.30 \%, 4.80 \%, 4.30 \%, 3.80 \%, 1.10 \%$, and $12.60 \%$, respectively.

The ctDNA mutational landscape of the samples, except for those with gene amplification and high microsatellite instability, in our study cohort is summarized in Figure 1. TP53 mutations constituted more than $16 \%$ of the detectable mutations. All the tumor types, except the ovary, kidney and apocrine gland tumors, harbored at least one type of TP53 mutation. Other common driver genes, such as KRAS and PIK3CA mutations, were responsible for an additional $10 \%$ of the mutations in the studied samples. Each cancer type showed a distinct mutational landscape, which indicated the importance of molecular profiling in cancer treatment.

Figure 2 shows the genes that were responsible for nonsynonymous mutations in NSCLC, breast cancer, stomach cancer, pancreatic cancer, colorectal cancer, and ovarian cancer with peritoneal carcinomatosis. Among 71 mutations in NSCLC, 16 (25.4\%) mutations were observed in the TP53 gene, 4 mutations were observed in the AR gene, 4 mutations were observed in the BRCA2 genes, 3 mutations were observed in the EGFR gene, and 3 mutations were observed in the GNAS gene, and these mutations in total accounted for approximately another $25 \%$ of the overall mutations. In breast cancer, PIK3CA was the most frequently mutated gene, accounting for one-quarter (9) of the 35 total genetic mutations in breast cancer samples, followed by ESR1 $(7,20 \%)$ and TP53 (3, 9\%). Similar to the case in NSCLC, most (5 mutations) of the 25 total mutations found in stomach cancer were in TP53. Mutations in ERBB2 $(4,16 \%)$ and ATM $(3,12 \%)$ were also frequently found in stomach cancer. Among 43 total mutations found in pancreatic cancer, PMS2 mutations $(8,19 \%)$ were the most frequent, followed by mutations in the KRAS $(7,16 \%)$ and MET 
$(3,7 \%)$ genes. RET and TP53 mutations were the most frequent in the 33 total mutations observed in colorectal cancer $(4,12 \%)$, followed by mutations in the AR, BRCA2 and GNAS genes $(2,6 \%)$. Among the 17 mutations identified in uterine cancer, TP53 $(7,41 \%)$ and KRAS $(2,12 \%)$ mutations made up more than half. In ovarian cancer with peritoneal carcinomatosis, more than one mutation was noted in the PIK3CA $(3,16.67 \%)$ and TP53 $(2,11.11 \%)$ genes.

Table 3 shows the cases with specific shared genetic mutations. KRAS (G12D) mutation was observed in five patients with pancreatic cancer. Mutations in GNAS (R201H), TP53 (G244C), ESR1 (D538G), TP53 (R175H), PDGFRB (L724P), KRAS (G12D), PMS2 (H479Q), and PMS2 (K413R) were observed in more than 2 cases in the respective cancer types. Except for the particular mutations listed in Table 2, approximately $94.7 \%$ of the remaining mutations were so unique that there was no duplication among the cases.

Figure 3a shows the extent of gene amplification and tumor mutation burden (TMB) observed in all types of cancer in this study. TMB is generally defined as the number of mutations in a tumor cell. We characterized TMB as low or high using a cutoff value of 6 nonsynonymous mutations per observed gene set, which is equivalent to 10 mutations per million base pairs, based on the $75^{\text {th }}$ percentile of data (Figure $3 b$ ). We observed that approximately $23.6 \%$ (21 out of 68 nonsynonymous mutations) of the tumors exhibited high TMB. Most of the patients with high TMB were patients with breast and colorectal cancers ( $n=4$, respectively), followed by patients with lung cancer and biliary cancer ( $n=2$, respectively). Figure $3 \mathrm{c}$ demonstrates the cancer types with high TMB and their respective number of mutations. Immune checkpoint inhibitors are clinically effective in tumors with high TMB.

\section{Case study reports}

We also examined two cases with clonal mutations after standard cancer treatment and dendritic cell immunotherapy. The first case included a 58-year-old woman diagnosed with stage IV pancreatic cancer with metastasis to the lymph nodes. Her pancreatic cancer was first diagnosed in April 2019. She started S-1, an oral 5-fluorouracil (5-FU) prodrug, chemotherapy in August and received subsequent radiotherapy (35 Gy in 5 fractions) in October. She underwent a full course of dendritic cell (DC) immunotherapy with adjuvant S-1 monotherapy from April 2020 to July 2020. During the follow-up in August 2020, her contrast-enhanced computed tomography (contrast CT) showed regression of the primary tumor, and the patient achieved partial response (PR). In October 2020, another contrast CT exam showed metastasis to the lymph nodes and was categorized as having stable disease. Her pre- and postDC therapy assessments showed that there was little alteration in the tumor clone, and the circulating tumor DNA titers were almost unchanged. However, there was a new clonal mutation in the ESR1 gene after treatment, which was consistent with the stable disease categorization. A summary of her liquid biopsy results is displayed in Table $4(A)$ and (B). 
The second case included a 38-year-old woman diagnosed with triple-negative breast cancer (TNBC). She underwent total mastectomy with reconstructive surgery in May 2020. She received combination therapy of anthracyclines and paclitaxel in July 2020, followed by paclitaxel from October to December. A full course of DC immunotherapy was given between May and October 2020. She was clinically categorized as having stable disease. Her pretreatment liquid biopsy results showed a high level of circulating tumor DNA, with a TP53 mutation being the major clonal mutation. TP53 mutation is associated with chemotherapy resistance and partly responsible for the poor prognosis of TNBC. After surgery, chemotherapy and DC immunotherapy, the patient's circulating tumor DNA level of dominant oncogenic clones dramatically decreased. In addition, a new clonal mutation was detected in the PMS2 gene. Table 5 (A) and (B) show a summary of her liquid biopsy results.

\section{Discussion}

In our study cohort, all the circulating free DNA samples had at least one detectable somatic mutation. Nonsynonymous mutations, that is, mutations involving amino acid changes, were the most frequently observed mutation type in our study. More than $90 \%$ of the patients showed at least one nonsynonymous mutation in their circulating free DNA. In terms of mutational landscape, the most frequently mutated gene was the tumor suppressor gene TP53, and the next most frequently mutated genes were driver genes such as PIK3CA and KRAS. These findings were consistent with the Pan-Cancer Cohort study, which analyzed the genomic profiles of thousands of tumor biopsies and matched normal tissues [14].

We found that approximately $94.7 \%$ of the mutations were unique to each patient; that is, the mutations were not shared between any two patients. A study of 1059 patients with NSCLC from The Cancer Genome Atlas (TCGA) reported that the percentage of overlapping mutated genes between any patient pair was less than $10 \%[15]$.

Tumors containing a high mutational burden (TMB) constituted approximately a quarter of all the tumors we studied. Different cancer types showed different TMBs. Previous studies reported that tumors with high blood TMB can benefit from neoantigen-targeted antitumor responses and are susceptible to immune checkpoint inhibitors $[16,17]$. Recently, the NCCN guidelines noted that dynamic changes in tumor mutational burden (TMB) may be a helpful biomarker in selecting patients for immunotherapy.

According to The Cancer Genome Atlas (TCGA), PIK3CA is the most frequently mutated gene in both luminal A and B breast cancer (45\% and 29\%, respectively), whereas the TP53 gene shows the highest frequency of mutation ( $80 \%$ ) in TNBC according to an analysis of the genomic profiles of tumor biopsies from 463 breast cancer patients [18]. In one ctDNA-based study of 10,000 pancancer Chinese patients, PIK3CA was the most frequently mutated gene, and PIK3CA mutations were identified in $39.1 \%$ of breast cancer samples [19]. Similarly, we found that $26 \%$ of the breast cancer samples harbored PIK3CA mutations in our study. Additionally, we observed that TP53 was the major gene involved in the case of a 38-year-old woman with TNBC. Therefore, our liquid biopsy result was similar to previous studies on genomic profiling of tissue and liquid biopsies in this regard. Since there is almost no effective targeted 
therapy for TP53 and KRAS mutations, identification of these mutations could help identify candidates for targeted immunotherapies.

Clonal evolution was observed in two of the patients who had undergone genomic profiling just before or after undergoing chemotherapy, radiotherapy and immunotherapy. This is proof that liquid biopsy can track tumor dynamics in real time and can be useful for determining prognosis as well as valuable in treatment evaluation. Liquid biopsy can also provide critical information for developing personalized dendritic cell immunotherapy using tumor-specific neoantigens in accordance with clonal evolution within a few months. Therefore, liquid biopsy can be a solution to track clonal changes for the selection of effective therapies.

This study has a few limitations. The number of mutations that we could detect from liquid biopsy was fewer than that from tumor biopsy. On the other hand, we cannot draw universal conclusions based on this small sample cohort. Larger studies have also reported similarities and discrepancies between these two methods and postulated that intratumoral heterogeneity and clonal evolution might also be responsible for such discrepancies. In addition, the detection sensitivity depends on individual variations, such as tumor burden and disease stage [20]. Despite the above limitations, this study indicates the importance of tailoring tumor-specific antigen-based immunotherapy in accordance with the liquid biopsy result in each treatment stage.

\section{Conclusions}

Nonsynonymous mutations were the most frequently encountered genetic mutations in somatic tumors. Tumor mutations were unique to each patient. Common mutations were limited among the different types of cancer. Clonal evolution was observed in patients just before or after receipt of chemotherapy, radiotherapy and immunotherapy. These findings contribute to the emerging evidence that liquid biopsy can replace tissue biopsy in treatment selection and evaluation and monitoring of disease progression. Liquid biopsies can also serve as a rapid, cost-effective and noninvasive alternative to tumor biopsies for tumor-specific antigen-based immunotherapy despite limitations in detection sensitivity. However, further experimental and interventional studies are required to investigate the immunogenicity of tumor-specific antigens from liquid biopsy to ensure that the strategy is practical for immunotherapy.

\section{Declarations}

Acknowledgments

We would like to acknowledge Ms. Kumi Kimura and Ms. Hanano Sakurai for compilation of the relevant data for this study. 
KZYM (ORCID: 0000-0001-5553-1245) was involved in data analysis and interpretation and drafted the manuscript.

JT is the principal investigator and corresponding author, who conceptualized or designed the entire study, conducted data analysis and revised the manuscript.

MS is the coinvestigator, who contributed to the conceptualization and implementation of the study and revision of the manuscript.

$\mathrm{RH}, \mathrm{MK}$ are the coinvestigators of the study.

YS contributed to the design and implementation of the study.

Additional Information (including a Competing Interests Statement)

The authors declared no conflict of interest.

\section{References}

1. Nakamura, Y. et al. Clinical utility of circulating tumor DNA sequencing in advanced gastrointestinal cancer: SCRUM-Japan GI-SCREEN and GOZILA studies. Nat Med. 2020; 26, 1859-1864.

2. Sunami, K. et al. Feasibility and utility of a panel testing for 114 cancer-associated genes in a clinical setting: A hospital-based study. Cancer Sci. 110(4), 1480-1490 (2019).

3. Cohen, J. D. et al. Detection and localization of surgically respectable cancers with a multianalyte blood test. Science. 23, 359(6378):926-930 (2018).

4. $\lrcorner$ Rolfo, C. et al. IASLC statement paper: liquid biopsy for advanced non-small-cell lung cancer (NSCLC). J Thorac Oncol. 13, 1248-68 (2018).

5. Fusco, M. J., West, H., Walko, C.M. Tumor mutation burden and cancer treatment. JAMA Oncol. 7(2), $316(2021)$.

6. Kim, S. T. et al. Prospective feasibility study for using cell-free circulating tumor DNA-guided therapy in refractory metastatic solid cancers: An interim analysis. JCO Precis Oncol. 1, 1-15 (2017).

7. Newman, A. et al. Integrated digital error suppression for improved detection of circulating tumor DNA. Nat Biotechnol. 34, 547-555 (2016). 
8. Schmitt, M. W. et al. Detection of ultrarare mutations by next-generation sequencing. Proc Natl Acad Sci USA. 109, 14508-1451 (2012).

9. Daan, C.et al. Technical validation of Roche AVENIO sequencing platform for liquid biopsies [abstract]. In: Proceedings of the American Association for Cancer Research Annual Meeting 2019, 2019 Mar 29-Apr 3, Atlanta, GA. Philadelphia (PA): AACR, Cancer Res. 79(13 Suppl): Abstract nr 1381 (2019).

10. Verma, S. et al. Analytical performance evaluation of a commercial next generation sequencing liquid biopsy platform using plasma ctDNA, reference standards, and synthetic serial dilution samples derived from normal plasma. BMC Cancer. 20, 945, https://doi.org/10.1186/s12885-020-07445-5 (2020).

11. Gu, Z. Complex heatmaps reveal patterns and correlations in multidimensional genomic data. Bioinformatics. 9 (15), 32(18):2847-9. doi: 10.1093/bioinformatics/btw313 (2016).

12. Kim, D. L., Lauren, H., Stephen, A. C., Deepa R. \& Nadine, T. When should tumor genomic profiling prompt consideration of germline testing? J Oncol Pract.15:9, 465-473 (2019).

13. Li, M.M. et al. Points to consider for reporting of germline variation in patients undergoing tumor testing: a statement of the American College of Medical Genetics and Genomics (ACMG). Genet Med. 22, 1142-1148 (2020).

14. Kandoth, C., McLellan, M., Vandin, F. et al. Mutational landscape and significance across 12 major cancer types. Nature. 502, 333-339 (2013).

15. Jia, Q. et al. Tracking neoantigens by personalized circulating tumor DNA sequencing during checkpoint blockade immunotherapy in non-small cell lung cancer. Adv Sci. 7(9):1903410. doi: 10.1002/advs.201903410 (2020).

16. Wang, Z. et al. Assessment of blood tumor mutational burden as a potential biomarker for immunotherapy in patients with non-small-cell lung cancer with use of a next-generation sequencing cancer gene panel. JAMA Oncol. 5, 696-702 (2019).

17. Gandara, D. R. et al. Blood-based tumor mutational burden as a predictor of clinical benefit in nonsmall-cell lung cancer patients treated with atezolizumab. Nat. Med. 24, 1441-1448 (2018).

18. Comprehensive molecular portraits of human breast tumors. Cancer Genome Atlas Network. Nature. 10, 490(7418):61-70 (2012).

19. Zhang, Y. et al. Pancancer circulating tumor DNA detection in over 10,000 Chinese patients. Nat Commun. 12, 11 (2021). 
20. Newman, A. M. et al. An ultrasensitive method for quantitating circulating tumor DNA with broad patient coverage. Nat Med. 20(5):548-54 (2014).

\section{Tables}

Table 1: Summary of demographic, clinical and pathological characteristics of the study cohort Characteristics

Guardant360

$$
(n=53)
$$

Age, n (\%)

$30 \mathrm{~s}$

$40 \mathrm{~s}$

$50 \mathrm{~s}$

$60 \mathrm{~s}$

$70 \mathrm{~s}$

$80 \mathrm{~s}$

$90 \mathrm{~s}$

Sex

Male

Female

Testing frequency

One time

Two times

Three times

Cancer diagnosis

First time

Recurrence

Metastases

No metastasis

One site

Multiple sites
49

1

49
3
1

$(92 \%)$

$(2 \%)$

38

38
15

(72\%)

(28\%)

\begin{tabular}{lc}
\hline No metastasis & 7 \\
One site & 14 \\
\hline Multiple sites & 32
\end{tabular}

7
14
32

(13\%)

(26\%)

$(60 \%)$
(Range: 32 - 76)

$$
(n=32)
$$

(Range: 35 - 91)

$\begin{array}{cccc}3 & (6 \%) & 2 & (6 \%) \\ 8 & (15 \%) & 0 & (0 \%) \\ 12 & (23 \%) & 7 & (22 \%) \\ 11 & (21 \%) & 10 & (31 \%) \\ 19 & (36 \%) & 9 & (28 \%) \\ 0 & (0 \%) & 3 & (9 \%) \\ 0 & (0 \%) & 1 & (3 \%)\end{array}$

31

(58\%)

16

(50\%)

(42\%)

16

(50\%)

23

(72\%)

(6\%) 9

(28\%)

0

(0\%)

30

(94\%)

2

(6\%)

6

(19\%)

7

19

(59\%) 
Table 2: Summary of Guardant Health Genomic Profiling

Circulating free DNA (cfDNA) in 20 milliliters of blood (mean \pm SD)

: $162.7 \pm 352.3$

ng

Proportion of circulating tumor DNA (ctDNA) among cfDNA

: $0.006 \%-$

Patients with presumed germline pathogenic variant $(n, \%)$ $90.6 \%$

Number of mutations per patient, except gene amplification and MSI-high (range, : 0 - 21 , mean $\pm \mathrm{SD}$ )

Nonsynonymous substitution per sample (range, mean \pm SD)

$5.6 \pm 4.3$ $: 0-14$,

Patients with nonsynonymous substitution (n, \%) $3.6 \pm 2.8$ : $90,90.1 \%$

Other types of mutation (compared to total number of mutations)

Synonymous substitution

: $16.30 \%$

Frameshift mutation

: $4.80 \%$

Nonsense mutation

: $4.30 \%$

Mutation in 5'- and 3'-UTRs (untranslated regions)

: $3.80 \%$

Partial deletion, fusion, duplication

: $1.10 \%$

Gene amplification, MSI-high

: $12.60 \%$

SD: Standard deviation

Table 3: Duplicated cases observed with particular genetic mutation

\begin{tabular}{llc}
\hline Type of cancer & Mutated gene & Number of patients \\
\hline Non-small-cell lung cancer & GNAS(R201H) & 2 \\
\hline (NSCLC) & TP53(G244C) & 2 \\
\hline Breast cancer & ESR1(D538G) & 2 \\
\hline Biliary cancer & TP53(R175H) & 2 \\
\hline Pancreatic cancer & PDGFRB(L724P) & 2 \\
\hline & KRAS(G12D) & 5 \\
\hline & PMS2(H479Q) & 2 \\
\hline
\end{tabular}


Table 4. Summary of the genetic profile of 58-year-old woman diagnosed as stage IV pancreatic cancer with metastases to the lymph nodes: before and after undergoing cancer treatments

A. Baseline results

Type of mutation

Gene Amino acid alternation

Frequency in cfDNA

Nonsynonymous

chr13:28049456 T>C

Nonsynonymous

FLT3

Arg322GLy

$0.03 \%$

chr13:32336753 G>T

Synonymous

chr1:156875584 C>T

Synonymous

BRCA2

Gly800Cys

$0.08 \%$

NTRK1

Gly473Gly

$0.06 \%$

chr2:47414292 G>A

Synonymous

chr3:10149950 A>G

Synonymous

MSH2

Ala272Ala

$0.05 \%$

VHL

Gln209Gln

$3.19 \%$

FBXW7

Asn572Asn

$0.08 \%$

chr4:152324323 A>G

$B$. After undergoing cancer treatments

\begin{tabular}{cccc}
\hline Type of mutation & Gene & Amino acid alternation & Frequency in cfDNA (cfDNA concentration) \\
\hline $\begin{array}{c}\text { Nonsynonymous } \\
\text { chr6:152098782 C }>\text { A }\end{array}$ & ESR1 & P535H & $0.03 \%$ (0.05 ng) \\
\hline Type of mutation & Gene & Amino acid alternation & Frequency in cfDNA \\
\hline $\begin{array}{c}\text { Synonymous } \\
\text { chr10:8796510 A }>\text { C }\end{array}$ & PTEN & T350T & $0.08 \%$ \\
Synonymous & VHL & Q209Q & $3.18 \%$ \\
\hline
\end{tabular}

Table 5. Summary of the genetic profile of 38-year-old woman diagnosed with triple negative breast cancer: before and after undergoing cancer treatments

A. Baseline results

\begin{tabular}{|c|c|c|c|}
\hline Type of mutation & Gene & Amino acid alternation & $\begin{array}{c}\text { Frequency in cfDNA } \\
\text { (cfDNA concentration) }\end{array}$ \\
\hline $\begin{array}{c}\text { Nonsynonymous } \\
\text { chr17:7675088 C>T }\end{array}$ & TP53 & $\mathrm{R} 175 \mathrm{H}$ & $4.59 \%(1.74 \mathrm{ng})$ \\
\hline Type of mutation & Gene & Amino acid alternation & Frequency in cfDNA \\
\hline $\begin{array}{c}\text { Nonsynonymous } \\
\text { chr4:56110468 G }>\mathrm{C}\end{array}$ & KDR & T397S & $2.75 \%$ \\
\hline $\begin{array}{c}\text { Nonsynonymous } \\
\text { chr3:12604282 G>T }\end{array}$ & RAF1 & $\mathrm{H} 230 \mathrm{~N}$ & $0.08 \%$ \\
\hline $\begin{array}{c}\text { Synonymous } \\
\text { chr2:47412509 C>A }\end{array}$ & MSH2 & G247G & $0.10 \%$ \\
\hline \multicolumn{4}{|c|}{ B. After undergoing cancer treatments } \\
\hline Type of mutation & Gene & Amino acid alternation & Frequency in cfDNA (cfDNA concentration) \\
\hline $\begin{array}{c}\text { Nonsynonymous } \\
\text { chr7:5987328 G>C }\end{array}$ & PMS2 & H479Q & $0.04 \%(0.05 \mathrm{ng})$ \\
\hline Type of mutation & Gene & Amino acid alternation & Frequency in cfDNA \\
\hline $\begin{array}{c}\text { Nonsynonymous } \\
\text { chr2:29225529 A >G }\end{array}$ & ALK & L1035P & $0.03 \%$ \\
\hline $\begin{array}{c}\text { Nonsynonymous } \\
\text { chr6:152011730 C>G }\end{array}$ & ESR1 & L391 V & $0.04 \%$ \\
\hline $\begin{array}{c}\text { Synonymous } \\
\text { chr7:5987526 } \mathrm{T}>\mathrm{C}\end{array}$ & PMS2 & K413K & $0.10 \%$ \\
\hline $\begin{array}{c}\text { Upstream } \\
\text { chr5:1295099 T>C }\end{array}$ & TERT & $\mathrm{N} / \mathrm{A}$ & $0.03 \%$ \\
\hline
\end{tabular}




\section{Figures}

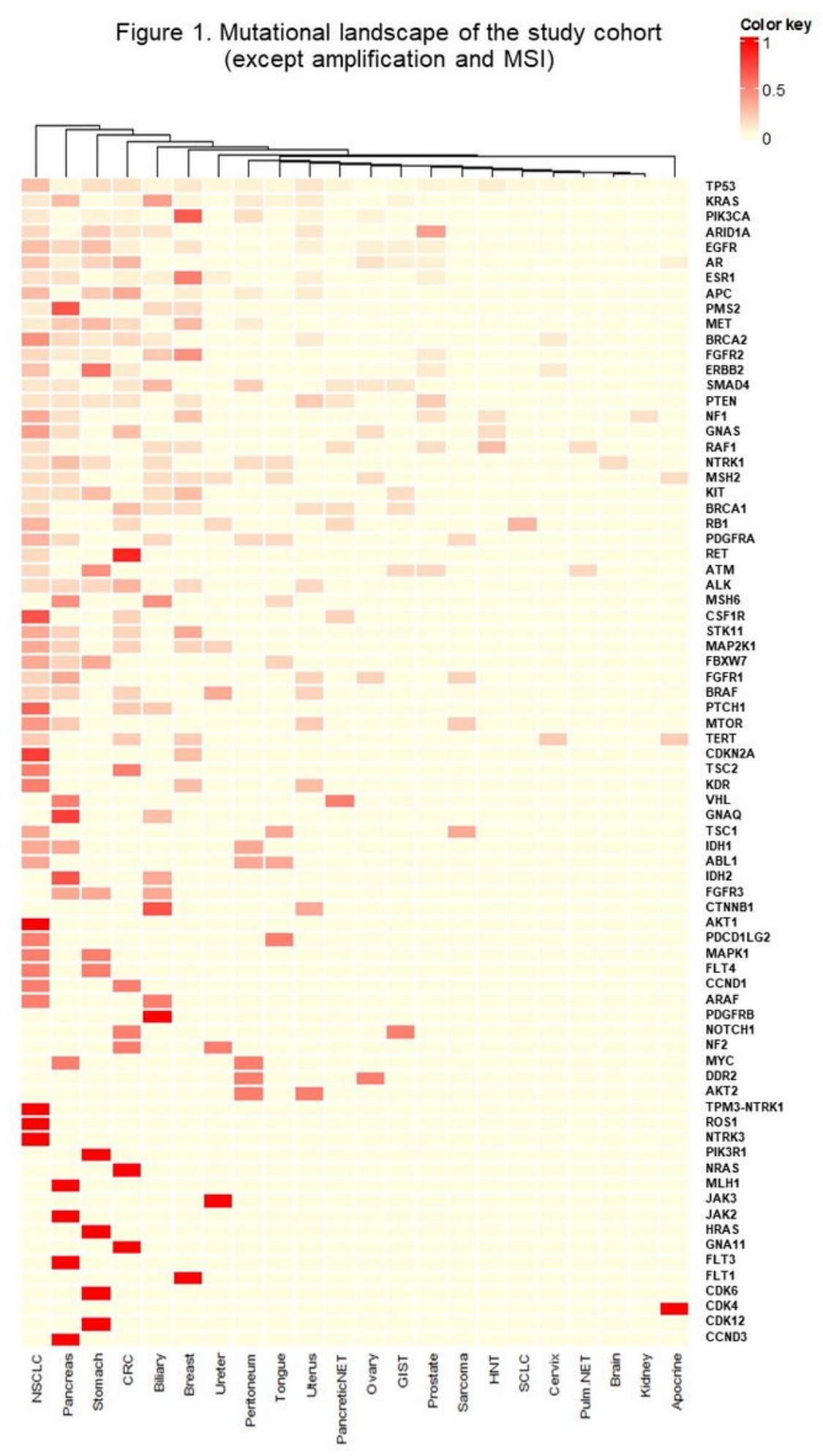

Figure 1

The most frequently mutated gene was the tumor suppressor gene TP53, and the second most frequently mutated genes were driver genes such as PIK3CA and KRAS. 
Figure 2. Genes that were responsible for nonsynonymous mutations
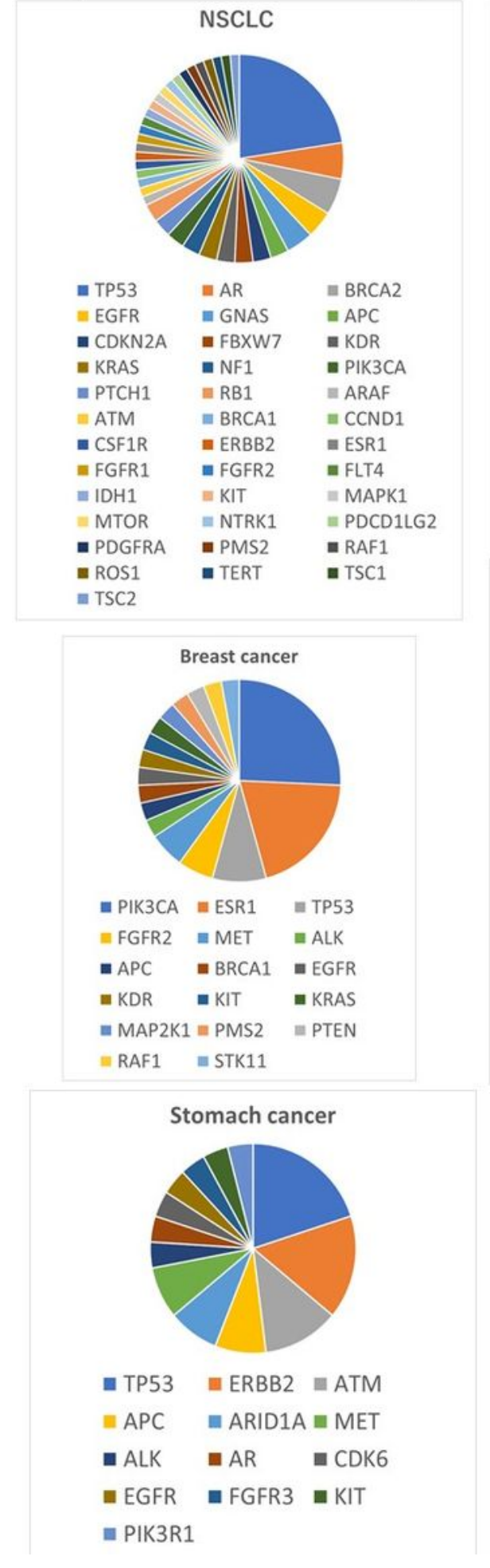

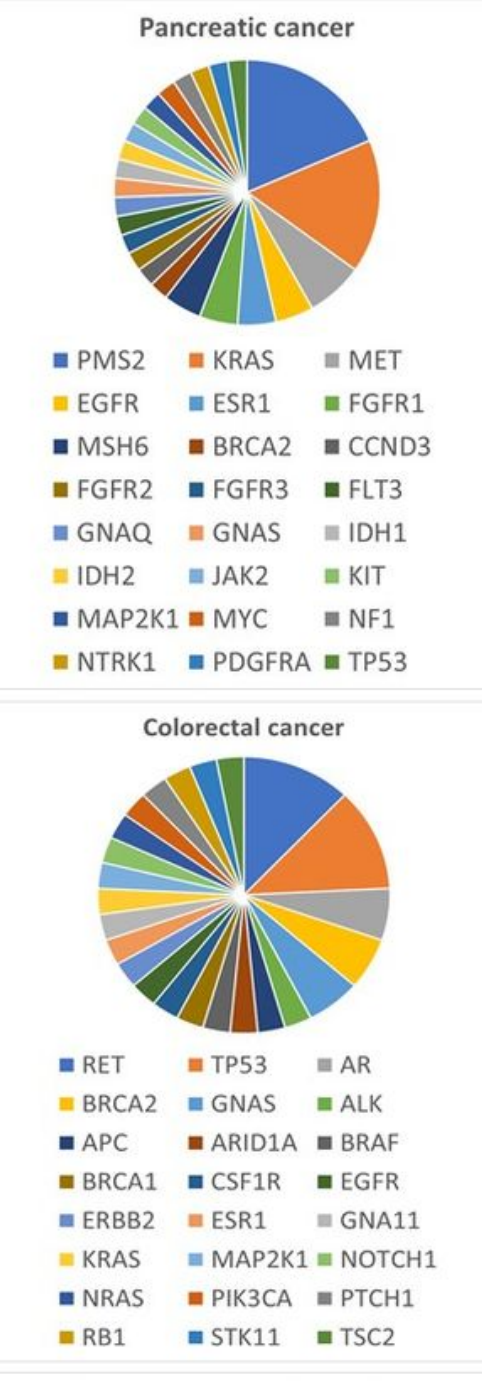

Ovarian cancer with peritoneal carcinomatosis
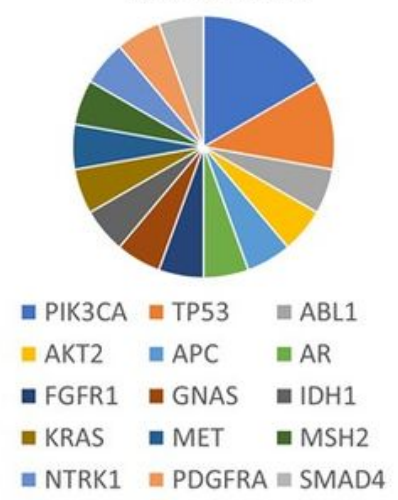

\section{Figure 2}

Among the genes that were responsible for nonsynonymous mutations in non-small-cell lung cancer (NSCLC), pancreatic cancer, breast cancer, colorectal cancer, stomach cancer, and ovarian cancer with peritoneal carcinomatosis, TP53 was one of the top three most frequently mutated genes, except in pancreatic cancer. 
Figure 3a. Extent of gene amplification and tumor mutation burden (TMB) in the study cohort

Extent of gene amplification

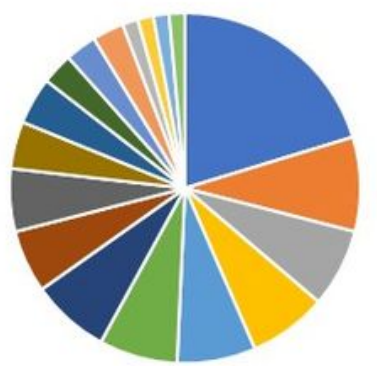

-EGFR =CCNE1 =BRAF "CDK6 =KRAS = MET

-MYC -FGFR1 -KIT - CCND1 - FGFR2 -ERBB2

$=$ PDGFRA =PIK3CA $=A R \quad$ "CCND2 $=$ CDK4 $=$ RAF1
Tumor mutation burden

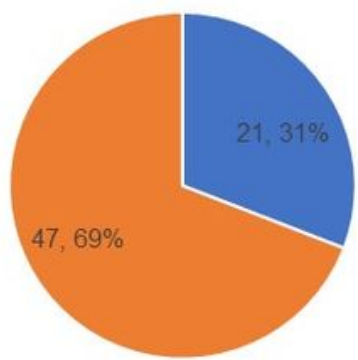

- $T M B \geqq 6 \quad-T M B<6$

Figure $3 b$. Distribution of tumor mutation burden (TMB) in all enrolled samples
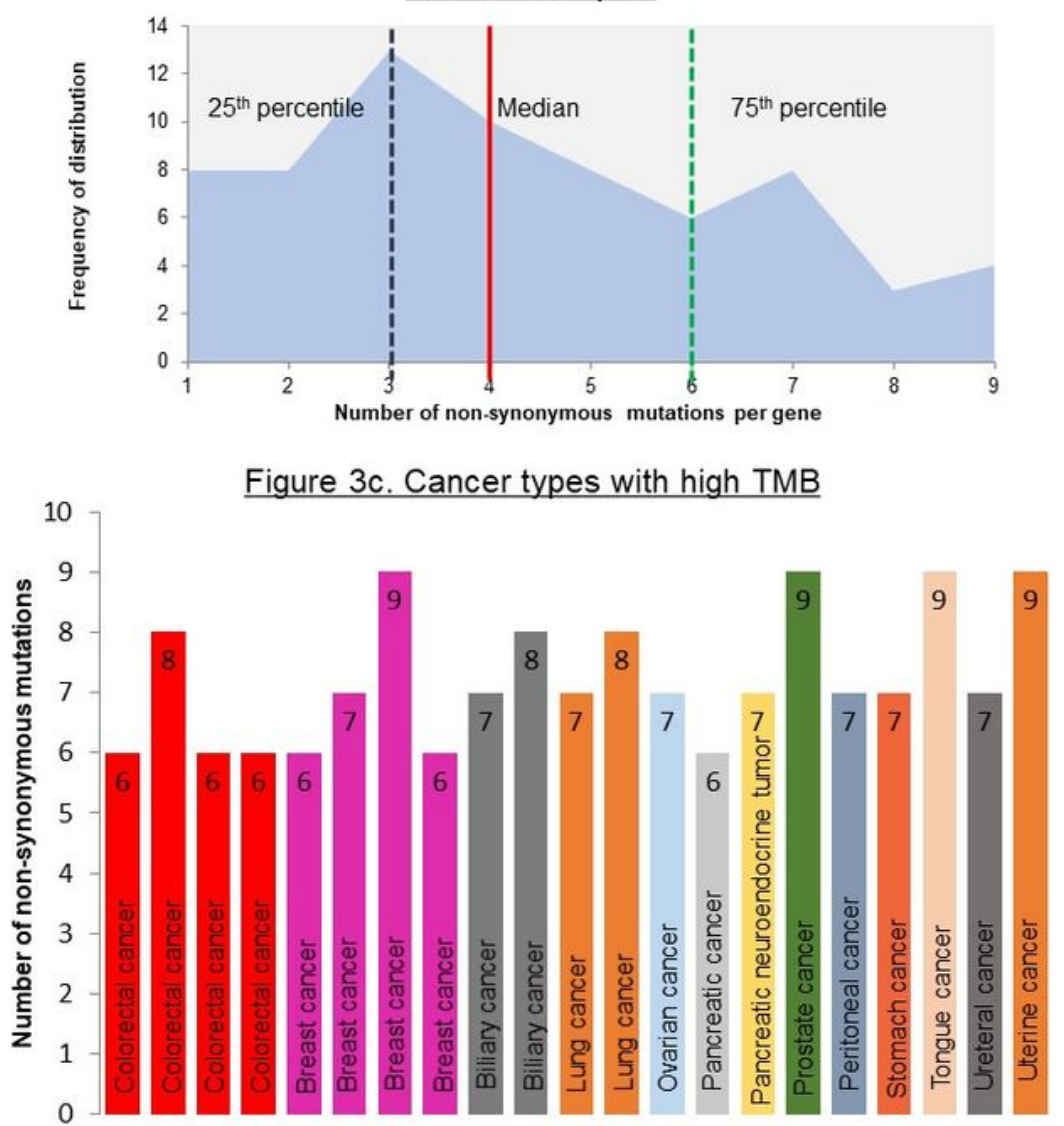

\section{Figure 3}

a. EGFR amplification was the most prevalent type in this study cohort. Approximately $23.6 \%$ (21 out of 68 nonsynonymous mutations) of the tumors exhibited a high tumor mutation burden (TMB). b. Vertical solid and broken lines indicate the median and upper/lower quartile values, respectively. Based on the upper quartile, the cutoff for high and low TMB was set at 6 mutations/mutated gene. c. Most of the 
patients with high TMB were patients with breast and colorectal cancers, followed by patients with biliary cancer and lung cancer (NSCLC).

\section{Supplementary Files}

This is a list of supplementary files associated with this preprint. Click to download.

- Supplementarydatafile1.pdf

- Supplementarydatafile2.pdf 